\title{
Numerical modeling of the dynamic strain aging in steels at high strain rates and high temperatures
}

\author{
R. A. Rubio ${ }^{1,2}$, M. Hokka ${ }^{1}$ \\ ${ }^{1}$ Impact - Multiscale Mechanics Research Group, Engineering Materials Science, Materials Science \\ and Environmental Engineering, Tampere University POB 589, FI-33014, Tampere, Finland E-mail: \\ mikko.hokka@tuni.fi \\ ${ }^{2}$ Department of numerical modelling, A3V Consulting S.L, C/ Gramil 9-1, Polígono Industrial P-29, \\ Collado Villalba 28240 Madrid, Spain. E-mail: ra.rubio@a3vconsulting.com
}

\begin{abstract}
Carbon steels can be heat treated to produce different microstructural variations and mechanical properties. At high temperatures the material plasticity and strength can be influenced by diffusional effects like the Portevin-Le Chatelier effect, leading to a commonly observed increased strength at elevated temperatures. The diffusional effects are influenced by the chemical composition, but also the heat treatment history that affects the local composition and especially the concentrations of free solute atoms. In this work, a numerical approach was implemented to reproduce the thermomechanical behaviour of two different microstructural variants of steel grade $\mathrm{C} 45$. The experimental data used to calibrate the model includes information of the plastic behaviour of material subjected to dynamic compression loading at a wide range of temperatures. Special emphasis was focused to describe the effects of the dynamic strain aging (DSA) on the flow stress. A strategy based on machine learning was implemented to obtain a model that reproduces the strengthening of the material due to diffusional effects. Cubic Support Vector Machine models were trained for both microstructure variants of the steel and different surfaces were obtained to describe the topology of the flow stress as function of temperature and strain rate. The model predictions were compared to the behaviour described by the Johnson-Cook model to estimate the influence of the DSA effect on the strength of the material at high strain rates and temperatures. Furthermore, the model quantifies how the microstructure affects the strength of the material and the strength of the DSA-hardening.
\end{abstract}

\section{Introduction}

It is well known that the plastic behaviour of metallic materials depends on temperature and strain rate. There are several industrial processes involving high strain rates and temperatures where prediction of the operating conditions is useful for accurate selection of process parameters considering the expected material responses. There are models that are widely accepted and used in the industry to predict the dependence of the flow strength on applied strain, temperature, and strain rate, such as the Johnson-Cook Model (JCM) [1]. However, 
there are complex phenomena that can arise under certain circumstances which modify the behaviour of the metals leading to significant discrepancies between the observed flow stress and the predicted tendencies. One good example is the diffusional process of solute atoms to the cores of dislocations pinning them and consequently leading to an increase of strength. This is widely known as Dynamic Strain Aging (DSA) or Portevin Le-Chatelier effect [2].

Some commercial steels are known to be sensitive to diffusional migration of interstitial solute atoms like Carbon and Nitrogen. Consequently, the behaviour of such materials can include serrated flow at low strain rates, thermal hardening, and even negative strain rate sensitivity. See that the JCM only accounts for continuous thermal softening and positive strain rate sensitivity, and therefore, the presence of DSA leads to very significant errors, as shown in the work of Saffarini for C45 steel [3]. Their work revealed that the model is only applicable bellow a critical temperature where DSA started to be noticeable. A previous work by Hokka et al. [4] reported that the microstructure has a strong influence on the strength of the DSA effect in C45 steel, showing that the diffusional effects had a greater influence in a globular microstructure with respect to a lamellar one. This observation is consistent with the reports of Brandaleze [5], which show that the dissolution of cementite during the heat treatment to obtain globular pearlite increases the amount of Carbon in solid solution. Furthermore, the work of Min et al. [6] showed that the amount of plastic deformation also influences the strength of the DSA effect, because the binding energy between Carbon atoms and dislocation cores is higher than the cementite's affinity to carbon. Thus, an increase in the dislocation density promotes the dissolution of cementite as dislocations draw carbon atoms towards them more strongly than the cementite particles, and in consequence the amount of available Carbon in solid solution increases. On the other hand, it has been demonstrated that the cementite dissolution due to plastic deformation depends on different microstructural features $[5,7]$, and therefore the strain dependence of DSA also seems to vary from one steel microstructure to another.

There is significant effort in the literature oriented to modelling of the DSA effect in metals, including numerical fitting approaches like the work of Devotta et al. [8], in which a polynomial decomposition of the JCM parameters is presented to account for the appearance of DSA. On the other hand, there are complex physically based constitutive models like the one presented by Voyiadjis et al. [9] who reported a microstructural-physically based model which accounts for the DSA effect. Other physically based models have been developed especially by Nemat-Nasser and co-workers, who set a modeling framework based on describing the flow stress as the sum of the athermal and thermal components of flow stress. The later strategy considers that the athermal contribution depends on the structure of the material, while the thermal component includes the dynamic effects in addition to the influence of microstructure. By means of the later framework the group of Nemat-Nasser developed models for different strain rate regimes and also describing the dynamic strain rate effects for various metals and alloys [10,11, 12].

One common feature in modelling complex plasticity phenomena is that every analysis can turn unpractical when approaching it from a fundamental point of view. Consequently, statistical modelling techniques are new tools utilized to recognize implicit patters of input data with no necessity of explicit description of phenomena. Such data driven approaches are widely known as Machine Learning and have been utilized by different authors to recognize and quantify the influence of strain rate and temperature on the plastic behaviour of diverse metallic alloys [13]. The work of Li et al. [14] reported the use of a modified Johnson-Cook model conjugated with an artificial neural network model to predict the flow stress of DP800 steel over a wide range of temperatures and strain rates.

In the present work a simple and accurate approach is presented to estimate the DSA contribution to the flow stress. This approach is presents the flow stress as the sum of two contributions: one related to forest dislocation interactions and a second one related to 
frictional interactions between dislocations and solute atoms [15, 16]. Consequently, a Support Vector Machine (SVM) model was trained with experimental data measured at different strain rates and temperatures to predict the overall behaviour of the flow stress. Additionally, a JCM was adjusted with experimental data measured at low temperature where the DSA effects can be neglected, considering such temperature range as adifusional. Then the contribution of the frictional interactions between interstitial atoms and dislocations were calculated by the difference between the overall behaviour described by the model and the adifusional JCM. The approach was applied to two microstructural variants of the C45 steel and their DSA contributions to the flow stress were compared from one another.

\section{Materials and methods}

The material studied in this work has been investigated previously, and the microstructural characterization and the mechanical behaviour are reported in detail in ref. [4]. Therefore, only a summary of the materials and the experiments are given here. Four variants of the $\mathrm{C} 45$ standard steel were prepared with different heat treatments, and two of them are considered in this study. The Reference (R) microstructure was acquired by austenization annealing followed by isothermal annealing below the austenization temperature. The globular pearlite (GP) structure was obtained by adding an additional annealing cycle to the reference heat treatment. SEM images of the microstructures, the phase fractions, and chemical composition are given in ref. [4]. In this work we use the results of the mechanical testing carried out at different strain rates and temperatures for the R and GP structures. The tests were carried out with a Split Hopkinson Pressure Bar device with high temperature capabilities. The exact description of the test equipment is given in references $[4,17,18,19]$.

\section{Numerical approach}

A common assumption in the literature is to consider that the flow stress comprise the two different components; one related to the dislocation forest interactions and the other related to frictional interactions between dislocations and solute atoms $[15,16]$. The modelling framework used in this work consist of a model that predicts the total overall flow stress and a second part model that estimates only the component related to dislocation forest interactions. Thus, the DSA contribution is estimated by the difference between the two mentioned models.

An SVM model was trained in MATLAB to predict the flow stress as a function of strain, strain rate, and temperature $\left(\sigma_{T}(\varepsilon, T, \dot{\varepsilon})\right)$. The measured strain, strain rate, and temperature data were considered as predictors and the experimental flow stress as the corresponding responses.

The JCM was utilized to estimate the material response in absence of DSA. The latter model assumes a multiplicative decomposition of the flow stress in three contributions. Firstly the work hardening contribution, secondly the thermal softening, and thirdly the strain rate dependence, as shown in the following Equation:

$$
\sigma_{\text {adiff }}(\varepsilon, T, \dot{\varepsilon})=\left(a+b \varepsilon^{n}\right)\left(1-\left(\frac{T-T^{r e f}}{T^{m}-T^{r e f}}\right)^{m}\right)\left(1-c \ln \left(\frac{\dot{\varepsilon}}{\dot{\varepsilon}^{r e f}}\right)\right)
$$

where $a, b, n, m$ and $c$ are the standard Johnson-Cook model parameters.

The calibration of the JCM was done using the stress strain curves obtained in a range of temperature bellow $800 \mathrm{~K}$. The reference conditions were a temperature of $298 \mathrm{~K}$ and a strain 
rate of $2000 \mathrm{~s}^{-1}$. The identification of the model parameters was decomposed in to two numerical optimization processes:

1. Identification of the parameters $a, b$, and $n$ by means of experimental data at reference conditions, for which Equation ( 1) reduces to $\sigma(\varepsilon, T, \dot{\varepsilon})=\left(a+b \varepsilon^{n}\right)$. This step was performed using the Levenberg-Marquardt algorithm to minimize the distance from the model predictions to the experimental data.

2. Identification of parameters $m$ and $c$ using the full experimental data set. This step was performed using a hybrid optimization approach to minimize the distance from the model predictions to the experimental data. Thus, the process initially uses the genetic algorithm implemented in MATLAB to find the neighbourhood of the global minimum in the search space. Afterwards, a local method is utilized to find the local minimum that is closest to the global algorithm solution, yielding the parameter combination that best fit the experimental data.

The parameters of the JCM model for both microstructures are shown in the Table 1.

Table 1: Computed JCM parameters for the R and GP configurations

\begin{tabular}{|c|c|c|c|c|c|}
\hline Microstructure & $\mathrm{a}$ & $\mathrm{b}$ & $\mathrm{n}$ & $\mathrm{m}$ & $\mathrm{c}$ \\
\hline $\mathrm{R}$ & 686 & 869 & 0.49 & 0.63 & 0.057 \\
\hline GP & 553 & 890 & 0.57 & 0.59 & 0.053 \\
\hline
\end{tabular}

Once the JCM was calibrated, the DSA components of the flow stress as a function of strain, strain rate, and temperature were computed by the difference $\sigma_{T}(\varepsilon, T, \dot{\varepsilon})-$ $\sigma_{\text {adiff }}(\varepsilon, T, \dot{\varepsilon})$.

\section{Results and discussion}

Figure 1 presents the flow stress as a function temperature and strain rate at different strains predicted by the SVM learning models for the R and GP microstructures. It is possible to see that the machine learning approach is capable in obtaining models that are in good agreement with the experimental data. Furthermore, the obtained behaviours are smooth and consistent for a wide range of strains.

The DSA contribution of the flow stress was calculated by the difference between the flow stress predicted by the SVM learning model and the JCM as shown in Figure 2 for the $\mathrm{R}$ and GP microstructures at $20 \%$ of strain and at strain rate of $4500 \mathrm{~s}^{-1}$. It is possible to see that the JCM does not account for the rise of the flow stress at high temperature due to the dislocation pinning by solutes atoms, while the SVM model can reproduce the material strengthening with increasing temperature. The difference between the two models is interpretated as the DSA component, and the maximum absolute values of the diffusive components in the R and GB microstructures were $310 \mathrm{MPa}$ and 295MPa, respectively. Even though the absolute value of the DSA contribution to the flow stress is slightly higher in the reference microstructure, the relative contributions of the DSA on the total flow stress for the two structures are $33 \%$ for the reference structure and $42 \%$ for the globular pearlite. 
Therefore, the globular pearlite structure seems approximately $9 \%$ more sensitive to DSA than the reference structure.
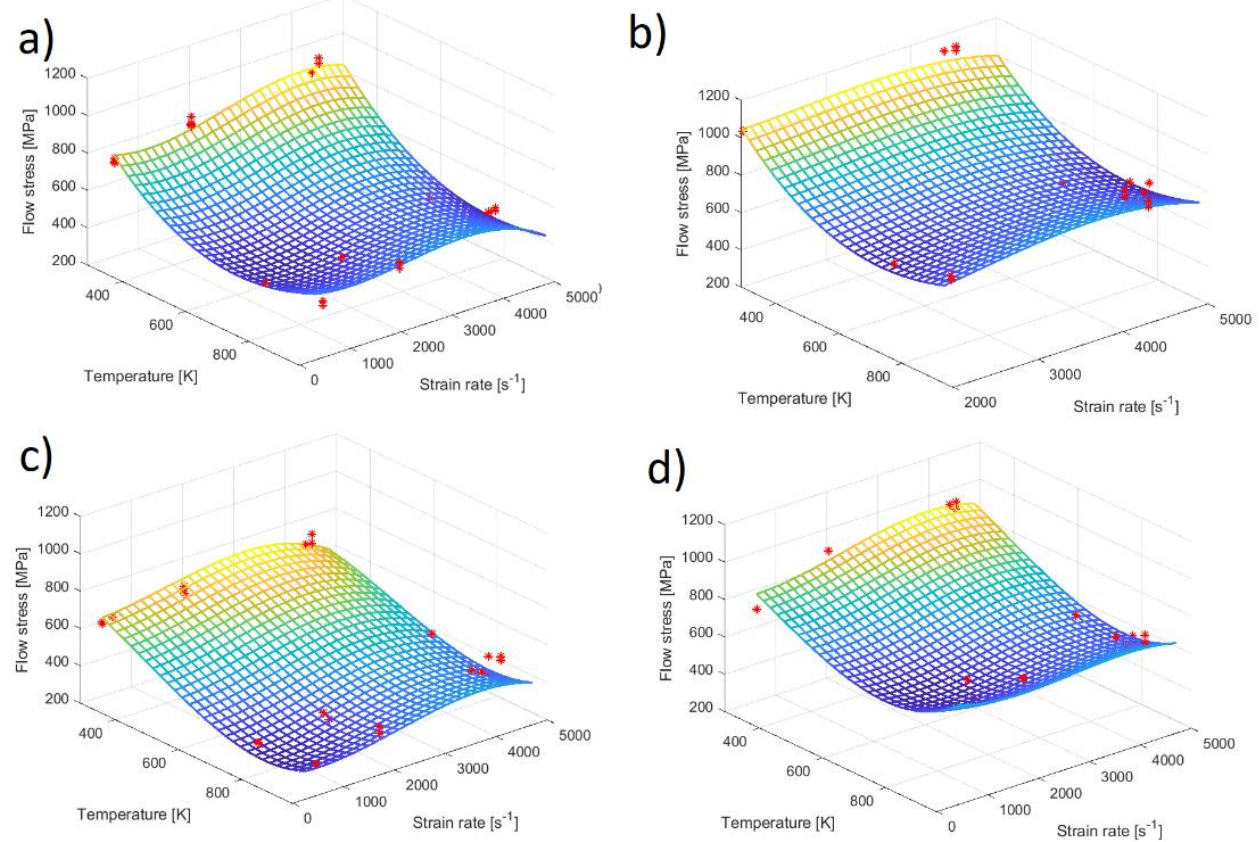

Figure 1 : Flow stress as a function of temperature and strain rate: a) $R$ structure at $2 \%$ strain, a) $R$ structure at 20\% strain, c) GP structure at 2\% strain, and d) G structure at $20 \%$ strain
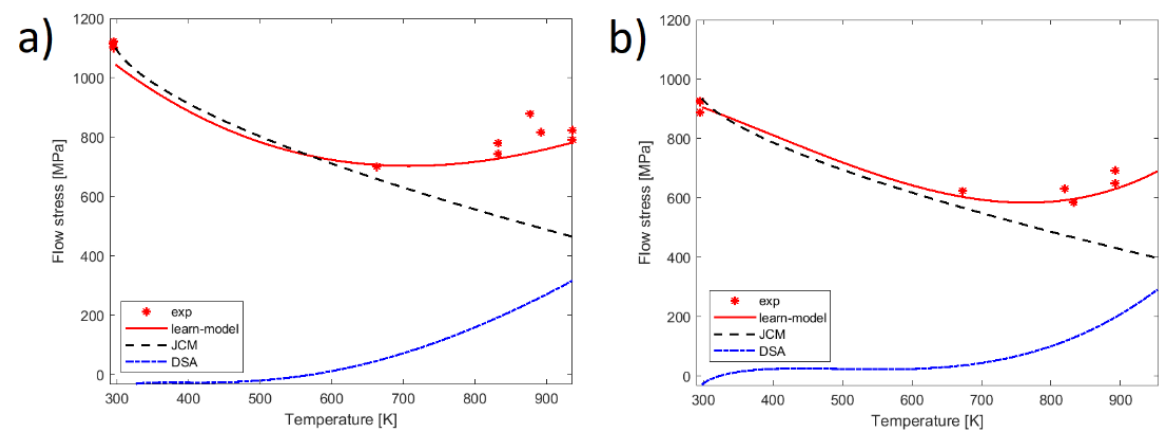

Figure 2: Flow stress as a function of temperature at $20 \%$ strain and $4500 \mathrm{~s}^{-1}$ predicted by the SVM model, the JCM, and the DSA component of the flow stress in a) reference structure and b) globular pearlite structure

\section{Conclusions}

A numerical approach was developed to estimate the contribution of the DSA effect on the flow stress of $\mathrm{C} 45$ steel at wide range of strain rates and temperatures. The methodology is based on an additive decomposition of the flow stress into two different contributions. First, a component describing the dislocation forest interactions, which is independent of the concentration of solute atoms and was modelled using the Johnson-Cook model. Second, the diffusive contribution related to the interactions between solute atoms and dislocations was 
computed by the difference between the predictions obtained with the Johnson-Cook model and a cubic SVM learning model used to interpolate the material behaviour between experimental data points.

The predicted behaviour obtained by the machine learning model is smooth and consistent with the empirically observed flow stress. Thus, it showed to be an accurate tool to reproduce the material behaviour in the range of strain rates and temperature bounded by the available experimental data. Such a tool can be readily used to generate constitutive information for finite element simulations.

The modelling approach used in this work also allows quantitative evaluation of the strength of the DSA effects in different microstructures. The maximum absolute strength (in $\mathrm{MPa}$ ) of the DSA effect is slightly higher for the reference microstructure. However, the relative contribution of the diffusive phenomena of the total flow stress is approximately $9 \%$ higher for the globular pearlite.

\section{References}

[1] G. R. Johnson and W. H. Cook, Proceedings 7th International Symposium on Ballistics, pp. 541-547, 1983.

[2] A. Portevin and H. Le-Chatelier, Trans. Am. Soc. Steel. Treat., vol. 52, pp. 457-478, 1924.

[3] M. H. Saffarini, Master Thesis American University of Sharjah, 2016.

[4] M. Hokka, J. Rämö, A. Mardoukhi, T. Vuoristo, A. Roth and V.-T. Kuokkala, J. Dyn. Behav. Mater. , vol. 4, pp. 452-463, 2018.

[5] E. Brandaleze, Procedia Materials Science, vol. 8, pp. 1023-1030, 2015.

[6] N. Min, W. Li and X. Jin, J. Mater. Sci. Technol., vol. 26, pp. 776-782, 2010.

[7] W. J. Nam, C. M. Bae, S. J. Oh and S.J. Kwon, Scripta mater, vol. 42, pp. 457-463, 2000.

[8] A. M. Devotta, P. V. Sivaprasad, T. Beno, M. Eynian, K. Hjertig, M. Magnevall and M., Metals, vol. 9, 2019.

[9] G. Z. Voyiadjis, Y. Song and A. Rusinek, Mechanics of Materials, vol. 129, pp. 352360, 2019.

[10] J. Chen and S. Nemat-Nasser, Acta Materialia, vol. 48, pp. 3131-3144, 2000.

[11] S. Nemat-Nasser, W. Guo and D. Kihl, J. Mech. Phys. Solids., vol. 49, pp. 1823-1846, 2001.

[12] S. Nemat-Nasser and W. Guo, Mat. Sci. Eng., vol. 284, pp. 202-210, 2000.

[13] D. P. Jang, P. Fazily and J. W. Yoon, Int. J. Plast., vol. 138, p. 102919, 2021.

[14] X. Li, C. C. Roth, D. Mohr, Int. J. Plast., vol. 118, pp. 320-344, 2019.

[15] A. Van Den Beukel and U.F. Kocks, Acta Metallurgica, vol. 30, pp. 1027-1034, 1982.

[16] A. Gilat and X. Wu, Int. J. Plast, vol. 13, pp. 611-632, 1997.

[17] M. Apostol, Ph.D. thesis, Tampere University of Technology, 2007.

[18] T. Leemet, M. Hokka, A. Shrot, M. Baeker and V.-T. Kuokkala, Mat. Sci. Eng. A, vol. 550, pp. 350-357, 2012.

[19] M. Hokka, T. Leemet, A. Shrot, M. Baeker and V.-T. Kuokkala, Experimental Mechanics, vol. 54, pp. 199-210, 2014. 